$(I R R=0.27, p<0.001)$ in months they were insured with Medicaid, versus individual- or employer-provided plans. Finally, the odds of experiencing a significant lapse in adherence was about two times higher $(O R=1.77, p=0.049)$ and lapse days occurred significantly more frequently $(I R R=1.38, p<0.001)$ in high financial strain months.

Conclusion Our results suggest that insurance coverage plays a vital role in ensuring patients maintain protective levels of PrEP over time. Although having any coverage is critical, our findings also show that those with private insurance may have more difficulty adhering to PrEP at protective levels, possibly due to restrictions these providers place on obtaining PrEP. Disclosure No significant relationships.

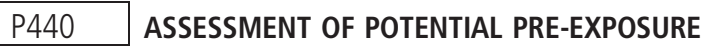 PROPHYLAXIS (PREP) COMPLIANCE IN INTRAVENOUS DRUG USERS ACCESSING OUTREACH SERVICES}

${ }^{1} J a n e$ Champion*, ${ }^{2}$ Kasey Claborn. 'University of Texas at Austin, Nursing, Austin, USA; ${ }^{2}$ University of Texas at Austin, Psychiatry, Austin, USA

10.1136/sextrans-2019-sti.525

Background Internationally, approximately one out of every ten new HIV infections has previously been attributed to intravenous drug use (IVDU). PrEP compliance among people with intravenous drug use (PWID) may decrease new HIV infection. This study assessed perceptions of PrEP, risk for contracting HIV, and potential compliance with PrEP among PWID.

Methods Clients accessing IVDU mobile outreach van services, aged $\geq 18$ years, completed anonymous self-report questionnaires about "Beliefs about Medicines" and "Perceived Risk of HIV" via iPad following informed consent.

Results Socio-demographics $N=100$, aged $18-63$ years $(50 \%$ $<37$ years), non-Hispanic White (56.5\%), Hispanic (38.4\%); male $(59.6 \%)$, sex with men (28\%), sex with women $(51 \%)$, sex with both (16\%), length of IVDU (1-9 years). Most separated/divorced (31.4\%) or never married (47.5\%); high school education or less $(54.5 \%)$; currently $(64 \%)$ or previously $(75.8 \%)$ homeless; ever prison $(54.5 \%)$ or arrested $(89 \%)$; HIV testing (98\%); currently HIV+ (2\%). Risk Behavior: Money/drugs for sex (50\%); uses condoms (30\%), alcohol (93\%), heroin (89\%), marijuana (95\%) prescriptions to get high (70\%), LSD (69\%), ecstasy (63\%) cocaine (89\%) meth (81\%), crack (59\%). Co-morbidities: Schizophrenia (7\%), Anxiety (59\%), Depression (60\%), Hepatitis (36\%), Diabetes $(6 \%)$ and HTN (25\%). Medication: Getting prescriptions easy (65.7\%); visiting doctor monthly/blood drawn no big deal (80.8\%); don't like PrEP for long time (55.6\%); concerned forget medications (53.6\%), or medication side-effects (42.7\%); think about HIV often (74.8\%); HIV risk moderate/ great (39.3\%). Most medications addictive (43.5\%); benefits out weight risks (57.6\%); safe $(47.5 \%)$; work better when taken regularly $(60.6 \%) ; 46 \%$ requested PrEP clinic information.
Conclusions PWID perceived high-risk for HIV. High levels of co-morbidities, substance use, limited HIV protective behavior and homelessness existed. Positive attitudes toward and interest in medication use for disease prevention and concerns regarding long-term medication or forgetting medications. PrEP adherence among PWID presents clinical challenges. Addressing risks, co-morbidities and inconsistent use of PrEP may optimize adherence.

Disclosure No significant relationships.

\section{P442 IMPLEMENTING PRE-EXPOSURE PROPHYLAXIS FOR HIV: EXPERIENCES IN A HEALTH DEPARTMENT BASED STI CLINIC}

${ }^{1}$ Andrea Lewis*, ${ }^{2}$ Candice Mcneil, ${ }^{3}$ Karen Hicks, ${ }^{4}$ Gregory Russell. ${ }^{1}$ Wake Forest Baptist Medical Center, Internal Medicine-Infectious Disease, Winston-Salem, USA; ${ }^{2}$ Wake Forest Baptist Medial Center, Internal Medicine-Infectious Disease, Winston-Salem, USA; ${ }^{3}$ Guilford County Health Department, PrEP Clinic, Greensboro, USA; ${ }^{4}$ Wake Forest Baptist Medial Center, Department of Biostatistics and Data Science, Winston-Salem, USA

\subsection{6/sextrans-2019-sti.526}

Background Despite the availability of PrEP, the uptake is suboptimal. Guilford County in North Carolina has seen a steady increase in the rates of HIV amongst high-risk groups, especially Black men-who-have-sex-with men (MSM). Many people receive preventative services through health departments and they are expected to play an integral role in PrEP services. The Guilford County Health Department (GCHD) PrEP clinic was established to provide services to their patients who were at high-risk of acquiring HIV. The aim of this study is to describe a health department's experience in implementing PrEP services and to identify risk related trends for patients screen/enrolled in an effort to optimize services.

Methods We conducted a retrospective review of six months of data from the GCHD PrEP clinic. Fisher's Exact Tests were used to test for differences between groups for outcomes of interest.

Results Between March 2018 and September 2018, 65 patients were referred to the PrEP clinic for initiation of PrEP. 94\% of all patients were male. Forty-eight patients identified as MSM of which $63 \%$ were Black. Of all patients screened at their referral visit for STIs, over half (55\%) tested positive for at least one bacterial STI, with pharyngeal gonorrhea being the most common $(p=0.022)$. Forty-six patients were prescribed PrEP, 31 were insured. 3 HIV seroconversions occurred in the interval between referral and initial PrEP visit.

Conclusion Majority of new HIV cases in Guilford County occur in Black MSM, and most of the patients screened for PrEP services at the clinic were Black MSM indicating that the patients accessing PrEP services are those that are most greatly impacted by the HIV epidemic in Guilford County. The high prevalence of STIs among patients initiating PrEP emphasizes the opportunity and significance of simultaneous STD screening and PrEP services. Financial resources have helped offer PrEP to high-risk groups. Disclosure No significant relationships. 